\title{
REFLEXIÓN CRÍTICA FRENTE AL NEUROSEXISMO
}

\author{
SONIA REVERTER-BAÑÓN ${ }^{1}$ \\ Universitat Jaume I
}

\begin{abstract}
RESUMEN: En el presente escrito se propone revisar los supuestos que guían la práctica neurocientífica al afirmar diferencias sexuales en el cerebro. Tras la constatación de que una gran parte de la investigación y publicación de hallazgos neurocientíficos dan por hecho tal diferencia, encontramos una gran carga de lo que se ha denominado neurosexismo. Como forma de superarlo y desde la teoría feminista y el compromiso político se propone un acercamiento crítico a las neurociencias, que a modo de colaboración reflexiva entre las disciplinas, pueda constituir un marco de superación de los prejuicios a la hora de hacer, pensar y diseñar la ciencia.
\end{abstract}

PALABRAS CLAVE: neurosexismo, diferencias sexuales, neurociencias críticas, neuroética, sexo/género, pseudociencia.

\section{Critical Thinking versus Neurosexism}

ABSTRACT: The aim of this paper is to offer a view of the assumptions that guide the practice of claiming sex differences in the brain. After the realization that much of the research and publication of neuroscientific findings assume such a difference, we found a great deal of what has been called neurosexism. As a way to overcome it and from a feminist theory with a political commitment we propose a critical approach to the neurosciences. This is understood as a reflective collaboration between disciplines that could provide a framework for overcoming prejudices in thinking and designing science.

KEY WORDS: neurosexism, sexual differences, critical neurosciences, neuroethics, sex/gender, pseudoscience.

\section{LAS CINCO ONZAS PERDIDAS}

En 1915, el famoso neurólogo Dr. Charles Dana ofreció sus conocimientos profesionales en el controvertido tema del voto de la mujer en las páginas de opinión del New York Times. El Dr. Dana expresó su preocupación de que «si las mujeres alcanzan el ideal feminista y viven como los hombres, incurrirán en el riesgo de demencia un 25 por ciento más de lo que tenemos ahora» (citado en Fine, 2010: 131). La base de esta opinión es que la mitad superior de la médula espinal, que controla la pelvis y las extremidades, es menor en las mujeres. Y ello, mantenía el Dr. Dana, afecta la eficacia de las mujeres en la evaluación de iniciativas políticas o de autoridad judicial. La participación política de las

1 Este trabajo se enmarca dentro del proyecto I+D «Política, empresa y educación desde la neuroética aplicada.» Ministerio de Economía y Competitividad (2014-2016). Referencia FFI2013-47136-C2-2-P financiado con fondos del MINECO. 
mujeres, es por ello, «peligrosa para su salud», afirmaba el Dr. Dana en dicho artículo periodístico.

De alguna manera, la concepción del Dr. Dana, refería a las teorías decimonónicas mantenidas por fisiólogos, anatomistas y biólogos que consideraban la inferioridad intelectual de las mujeres un hecho que tenía su explicación en la inferioridad de peso del cerebro femenino; exactamente cinco onzas menos. El fisiólogo darwinista George Romanes acuñó esto en 1887 como «las cinco onzas perdidas del cerebro femenino». En su estudio, publicado con el título de «Mental Differences Between Men and Women», Romanes afirma que es imposible decir cuándo las mujeres podremos equiparar nuestra potencia mental a la de los hombres, pero alerta que incluso en las mejores condiciones, y suponiendo que no haya ninguna evolución en el cerebro de los hombres, llevará muchos siglos "producir las cinco onzas perdidas del cerebro femenino" (Romanes, 1887: 666; citado en Wood, 2001: 172).

La cuestión es que las ciencias, y especialmente las relacionadas con la investigación del cerebro y las capacidades humanas, han sido bastante contundentes y obstinadas en mantener prejuicios en relación a la constitución biológica de las mujeres. El prejuicio patriarcal de la inferioridad racional de las mujeres no ha empezado a ser claramente contravenido por las ciencias hasta bien entrado el siglo $\mathrm{Xx}^{2}$. El siglo xIx produce un buen ejemplo del debate entre naturaleza y cultura sobre el tema de la diferencia (y desigualdad) de las mujeres. Precisamente de la mano de John Stuart Mill y Charles Darwin podemos entender los parámetros de este debate. Mill recalcó que lo que se llamaba «naturaleza de la mujer» es algo «eminentemente artificial», resultado de fuerzas de represión en muchas direcciones que acaban diciendo que lo que es natural es meramente "lo que es habitual». En ese sentido, y como afirma en su libro de 1869 The Subjection of Women, lo que es natural, en términos de mujeres y hombres, no es sino la ley de la fuerza, «un atavismo de un tiempo anterior a la ley moral». El libro de Mill, apareció dos años antes del libro The Descent of Man, and Selection in Relation to Sex (1871) de Charles Darwin. Como nos relata Boddice (2011: 323), Mill nunca hubiera imaginado una negación tan rotunda de su teoría igualitaria de la mano del científico más eminente de su tiempo, Darwin, quien acomodó las teorías de inferioridad de las mujeres a su teoría evolutiva. Darwin compartió con Mill una idea antiesencialista del ser humano, pero, sin embargo, expresó sin ambages (y a veces de manera contradictoria con ese anti-esencialismo que decía mantener) un pensamiento de prepotencia masculina en su teoría evolucionista desarrollada en The Descent cuando afirma en una de sus más citadas frases, «man has ultimately become superior to Woman». Con ello podemos decir que incorporó un prejuicio a una

2 Donna L. Maney analiza en un artículo de 2016 cómo la comunicación y la discusión pública de nuevos hallazgos referidos a la diferencia sexual de los cerebros está llena de «saltos lógicos y pseudociencia». Según afirma Maney, la razón es porque es un tema que «fascina a la vez que enciende al público» (Maney, 2016: 1). 
teoría científica, dándole así la preeminencia y la autoridad para convertirla en «verdad científica».

En esta misma línea argumentativa podemos entender que las palabras del Dr. Dana pueden ser catalogadas de «pensamiento pseudocientífico». Con esta etiqueta queremos referir, no al cúmulo de creencias y prejuicios no científicos mantenidos sobre la diferencia sexual por la opinión popular y/o personas y grupos no científicos; sino a aquellas creencias que de forma prejuiciosa y acientífica son mantenidas por parte de la misma comunidad científica. Precisamente, y como el sociólogo de la ciencia Nikolas Rose (1989, 1998, 2006, 2007) viene denunciando desde hace años, «el imperativo de hacer promesas exageradas acerca de su impacto, está dañando a la ciencia misma» (2014: 1). Las promesas, creencias y afirmaciones de la comunidad científica, como sabemos, revisten el problema adicional de poder ser utilizadas desde posiciones de autoridad científica, lo cual no sólo es perjudicial para la ciencia misma, como señala Rose, por contaminar el mismo conocimiento científico, sino para el conjunto de creencias culturales sobre el tema de la diferencia sexual que van a verse legitimadas, y van a justificar incluso decisiones institucionales y de políticas públicas en base a esas creencias (como ejemplo, el derecho al voto de las mujeres a principios de siglo xx). Precisamente el uso de la autoridad científica como principal argumento de una teoría es uno de los pilares fundamentales que tienen las teorías pseudocientíficas para justificar sus opiniones (Bunge, 1985: 71). Junto a ello estaría también la supresión y tergiversación de datos desfavorables a la opinión que se quiere legitimar como científica (Bunge, 1985: 71). De manera general podemos convenir con Thagard (1978: 228) que una disciplina o teoría pseudocientíficas satisface dos criterios: no muestra empeño en intentar evaluar la teoría en relación a otras y es selectiva a la hora de considerar otras afirmaciones.

Como Hansson (2015) nos explica, muchos autores han intentado ofrecer criterios de demarcación entre la ciencia y la pseudociencia, ofreciendo listas de criterios. Hansson nos ofrece una propuesta de aquellos aspectos que concitan un alto grado de acuerdo en los principales autores que han ofrecido propuestas de tales criterios: creencia en la autoridad como criterio para creer y aceptar lo que se afirma como verdadero o falso; imposibilidad de repetición de experimentos; ejemplos elegidos de forma que no son representativos de la categoría general a la que refieren; poca o nula voluntad de comprobación de la teoría; negación o desestimación de otras teorías o experimentos que entren en conflicto con la teoría; construcción de subterfugios para que la prueba de una teoría sólo pueda ser confirmada, no refutada, por el resultado; y, abandono de las explicaciones sin posible reemplazo, sin posibilidad de progresión teórica en el conocimiento aportado por las conclusiones de la teoría.

Podemos decir que la afirmación del Dr. Dana acerca de la diferencia sexual respecto a la médula espinal y sus repercusiones sobre la capacidad política de las mujeres cumple prácticamente estos siete criterios, por lo que podría servirnos de ejemplo claro de lo que es pseudo-ciencia. No hace falta, sin embargo, retroceder cien años para encontrar el mismo tipo de conclusiones 
pseudo-científicas acerca de las diferencias cerebrales entre hombres y mujeres. Este suele ser un campo minado de falsas creencias y suposiciones que contaminan constantemente el conocimiento científico; y de ahí la importancia de la demarcación entre conocimiento científico y pseudo-científico en el tema de las diferencias sexuales en el cerebro. Lo esencial de esta demarcación se vuelve obvio cuando constatamos que los argumentos científicos (y eventualmente también los pseudocientíficos) tienen no sólo repercusiones descriptivas, sino también normativas (Mahner, 2007). Por todas estas razones consideramos necesario hacer una revisión de las conclusiones a las que la comunidad científica pueda haber llegado en el tema de las diferencias sexuales en el cerebro. Poner al día las aportaciones neurocientíficas respecto a la posibilidad de seguir hablando de diferencias en el cerebro entre mujeres y hombres nos parece, a la vista de lo explicado hasta aquí, que no es una cuestión baladí, sino que comporta consecuencias vitales, en el sentido más literal de esta palabra.

Como la misma Roskies afirmó en su prominente artículo titulado «Neuroethics for the New Millenium», entender los mecanismos del cerebro en los comportamientos humanos tiene potencialmente «implicaciones dramáticas» para nuestra perspectiva sobre la ética y la justicia social (2002: 21). Resulta obvio el interés que para esta nueva disciplina ética reviste la investigación sobre las diferencias sexuales del cerebro. Para la ética práctica o ética de la neurociencia, como también Cortina nos propone (2010, 2011), el de las diferencias sexuales es un ejemplo a indagar en cuanto la práctica científica de las neurociencias se ve contaminada de forma constante de una mala praxis y cuyo origen denota una necesidad de reflexión y argumentación crítica sobre algunos presupuestos éticos (personales, pero también sociales y profesionales, como el ejemplo del Dr. Dana deja entrever). Para el segundo sentido, el de las implicaciones neurocientíficas (Roskies, 2002), o neurociencia de la ética (Cortina, 2010, 2011), es un ejemplo a explorar en cuanto una investigación ideologizada sobre las diferencias sexuales de los cerebros acarrea claramente unos presupuestos de diferencias morales (también sociales, culturales y políticas) entre hombres y mujeres que pueden alterar el mismo sentido de justicia social respecto a las expectativas y derechos de los diferentes sexos. Los peligros en ambos niveles de por sí ya legitiman el campo de estudio que se ha denominado «neuroética» y que propone un diálogo intenso entre profesionales de diversas disciplinas, así como entre éstos y el público y la gente común. El objetivo es poder encaminarnos a un «neuro-alfabetismo» (neuroliteracy) ${ }^{3}$ en el que puedan confluir los intereses de todos por el bienestar de los individuos y las sociedades. Uno de los objetivos de tal alfabetización sería, precisamente, la de «entender y críticamente juzgar» (Zardetto-Smith et al., 2002: 397) el material

3 El concepto de «neuroliteracy» proviene del concepto previo de «neuroroscience literacy», el cual, a su vez, proviene del de «science literacy». Se reconoce al texto de la Organización Mundial de la Salud (WHO) publicado en 2001 como el generador de esta preocupación por una alfabetización en las neurociencias. 
neurocientífico que aparece en los media, el cual, como hemos señalado, puede contener importantes errores de interpretación (Maney, 2016).

\section{Neuromitos y NEURoseXismo}

En ambos sentidos del término neuroética que acabamos de ver, y para el caso de las diferencias sexuales en el cerebro, las conclusiones pseudocientíficas a las que se ha llegado de manera consistente a lo largo de más de un siglo de investigación del cerebro denotan una falta de seriedad en la investigación y en sus conclusiones, que sólo puede ser atribuible a un conjunto de prejuicios culturales tan fuertes que incluso la misma ciencia se resiste a deshacerse de ellos. En este sentido cabría hablar de un «neuromito»; es decir, y como lo define la OCDE (2002) en su texto sobre neuromitologías, un malentendido, una mala interpretación o incluso una «deformación deliberada» de los hechos científicos con un propósito determinado (OCDE, 2002: 71; Pallarés-Domínguez, 2016).

La pregunta conveniente ahora es: ¿cuál es el propósito de afirmar una «deformación deliberada» respecto a un hecho científico? Sin duda esta pregunta nos lleva a profundizar tanto en la práctica científica como en las cuestiones ideológicas a las que la ciencia se pueda ver sometida, y ello es un tema de amplio recorrido y enorme calado. Vamos a intentar responder a esta pregunta delimitándola al ámbito de la afirmación de las diferencias sexuales en el cerebro; la cual, a nuestro parecer, es uno de los más importantes neuromitos en la historia de la investigación del cerebro. Para ello proponemos aclarar lo que entendemos que son los presupuestos más importantes que fundamentan las ideas que comúnmente gran parte de la comunidad científica sigue manteniendo cuando afirma que la diferencia sexual del cerebro es un hecho. Estas ideas las vamos a confrontar con la crítica que proclama que no hay base científica para confirmar tal diferencia. Para ello nos guiaremos, aunque no de manera exclusiva, por las investigaciones del grupo denominado NeuroGenderings Network con el interés de ayudar a separar la investigación científica sobre el cerebro de la pseudociencia sobre la diferencia sexual del cerebro ${ }^{4}$.

\footnotetext{
4 El NeuroGenderings Network es un grupo de trabajo originado a partir de un congreso organizado por el Center for Gender Research de la Universidad de Uppsala en 2010 con el título de NeuroGenderings: Critical Studies of the Sexed Brain. Dicho grupo aglutina disciplinas tales como neurociencias, humanidades, filosofía, ciencias sociales, estudios culturales, medicina, química, estudios de género y queer, teoría feminista y estudios de ciencia y tecnología. El objetivo es desplegar conceptos y debates más reflexivos para que puedan orientar mejor el diálogo entre las neurociencias y los intereses sociales y educativos (algo que esta red de trabajo denomina «neuropedagogía»). Muchas de las autoras críticas con el neurosexismo que se citan en el presente trabajo forman parte de esta red y han participado en todos o alguno de los encuentros señalados.
} 
El término neurosexismo (neurosexism) es un neologismo que precisamente reúne todos aquellos posicionamientos y teorías que utilizan la investigación neurocientífica para reforzar ideas prefijadas sobre las diferencias inherentes entre sexos. El término fue acuñado por la psicóloga Cordelia Fine en un artículo de 2008, aunque se popularizó a raíz de su libro Delusions of Gender: How Our Minds, Society, and Neurosexism Create Difference, de 2010. Con este término Fine refiere a las posturas sexistas, acríticas y acientíficas que basadas en una ideología patriarcal imperante pretende demostrar que el cerebro de mujeres y hombres es diferente simplemente por ser mujeres y hombres. Frente al neurosexismo aparece otro neologismo, neurofeminismo, que refiere precisamente a la respuesta crítica al anterior. El neurofeminismo pretende hacer una revisión crítica de las teorías científicas para desvelar cuánto de ideológico y cuánto de verdaderamente científico hay en las teorías sobre la diferencia sexual del cerebro.

El punto de partida es la constatación, como señala Fine (2008), de que las hipótesis sobre las diferencias entre los cerebros masculino y femenino se basan en resultados falsos, de mala calidad, malas metodologías, supuestos no probados y conclusiones prematuras. A ello se suma una apreciación insuficiente de la profundidad y alcance de los patrones culturales, creencias y expectativas en nuestras mentes, así como la constatación de una contaminación en las neurociencias por los prejuicios que guían la investigación de este campo concreto.

Veamos ahora las cuestiones que proponemos como las de más impacto a la hora de estudiar las diferencias sexuales en los cerebros.

\subsection{Confusión entre conceptos de sexo y género}

El género es reconocido como el principal factor de categorización social (Bem, 1983; Liben \& Signorella, 1980; Maccoby \& Jacklin, 1974). En general se considera al género como el principal elemento de continuidad de los roles, patriarcales o no, en la educación, en la cultura y en los diferentes procesos de socialización del individuo (Reverter-Bañón, 2010). Normalmente se analiza la división de género partiendo de la idea de que es la consecuencia de la dualidad de los sexos. Pero falta un acercamiento crítico que precisamente ponga en duda esa linealidad, ya que es asumida y no científicamente probada de manera evidente. Si hay una diferencia sexual biológica ello no implica de sí una construcción de género diferenciada; al menos no desde una visión crítica de las ciencias. La diferencia sexual del cerebro, si existe, no legitima ni nos direcciona de manera obvia hacia una construcción cultural, social y política diferenciada en roles distintos para los diferentes sexos. Es necesario un análisis de la investigación del sexo, en el sentido de explorar si hay una base científica que de sentido a separar los sexos (pre-social) en roles diferenciados y crianzas distintas (social). Sabemos que la diferenciación de género (social) de esos elementos es abrumadora. La cuestión será si esa diferenciación dual de los géneros que causa desigualdad y que se formula en base a la diferencia 
sexual tiene una base biológica que aconseje seguir diferenciando los géneros (y no sólo los sexos). Y esto es lo que la mayoría de estudios neurocientíficos no nos contestan.

Desde la teoría feminista (Lorber, 1993, 1996; Fausto-Sterling, 2000) se viene señalando desde hace décadas que ese mismo sustrato biológico es construido según categorías biológicas binarias que no siempre se muestran en los cuerpos de manera obvia, como nos aclara Medina-Vicent (2016). La variación entre sexos no es siempre discreta entre dos posibles modelos obvios (masculino o femenino); sino que los cuerpos ofrecen variaciones que pueden retar esa concepción dicotómica de la linealidad denominada «tres g» (genitales, gónadas, genes) y posibilitar una interpretación del sexo con más posibles modelos humanos (como Fausto-Sterling hace cuando nos propone cinco sexos; 2000), o entendida como un continuo (Joel, 2011; Joel et al., 2015).

El binarismo del sexo y del género conlleva ya de sí una concepción que va a tener implicaciones en el diseño mismo de las investigaciones, y por tanto, en los resultados. Estas consecuencias no intencionadas pueden distorsionar la verdad de un cuerpo, desnaturalizarlo, como en los casos de bebés intersexuales que son intervenidos para que se ajusten al modelo binario de los dos sexos. Para evitar ese esencialismo hay que diseñar las investigaciones identificando bien los aspectos específicos de interés de los conceptos de sexo y género (Johnson \& Repta, 2012; Runnels et al., 2014) y tratando de entender como buena práctica científica que el sexo/género es un proceso dinámico, multidimensional e interactivo (Springer, Stellman \& Jordan-Young, 2011).

Los conceptos de sexo y género son importantes porque remiten inexorablemente a los conceptos más amplios de biología y cultura (Haraway, 1990). En la bibliografía neurocientífica sobre las diferencias sexuales encontramos un baile conceptual y terminológico que revela por sí mismo la confusión respecto a los conceptos de sexo y género; y por tanto, a las posibilidades de conocer qué parte de la diferencia es natural y biológica y qué parte es social y construida. Así las diferencias entre mujeres y hombres (independientemente de si son neuronales o comportamentales) son a veces llamadas «diferencias de sexo» y a veces "diferencias de género». Kaiser (2012: 131) nos da ejemplos de esta confusión terminológica en los casos de importantes publicaciones neurocientíficas en revistas especializadas como Cerebral Cortex o Nature Neuroscience.

La literatura neurocientífica (y también de disciplinas relacionadas), como vemos, confunde constantemente los conceptos de sexo y género, usándolos a veces como sinónimos. La confusión es importante si pensamos que en la mayoría de los estudios en los que encontramos esta confusión son estudios que intentan revelar diferencias sexuales entre mujeres y hombres; es decir, son estudios que parten de una muestra que divide por géneros para medir diferencias de sexo. Esta confusión lejos de ser banal es indicativa de un desconocimiento conceptual que se despreocupa del alcance social y político que pueden tener las conclusiones de sus estudios. A la vez, confundiendo los conceptos o utilizándolos como sinónimos, demuestran normalmente que la conclusión a 
la que quieren llegar es de la que parten, que mujeres y hombres somos diferentes tanto en el género como en el sexo, ya que este último determina el primero.

Nuestra propuesta, y a la luz de los avances de la teoría feminista a este respecto, es que los conceptos de sexo y género son inseparables (Kaiser, 2012), que funcionan como una unidad (Fausto-Sterling, 2000; Haraway, 1990), y que por tanto la mejor opción de momento será referirnos a ellos con la fórmula sexo/género.

\subsection{Insuficiencia de evidencias, y prejuicios que guían las conclusiones.}

Como algunas voces destacadas en este debate han indicado (C.Vidal, 2011; Jordan-Young, 2010) y algunos estudios con metadatos ${ }^{5}$ confirman (Hyde, 2005, 2006, 2007), las supuestas evidencias científicas no nos llevan de forma científicamente probada a las conclusiones de que las diferencias sexuales están en el cerebro, y que es en el inicio de la formación del feto, gracias al circuito hormonal y a la definición genética y cromosomática que define la orientación sexual y la identidad de género. La cuestión es que tal proceso hormonal de la definición sexual no se ha demostrado que tenga implicaciones determinantes en la estructura o funcionalidad del cerebro (Jordan-Young, 2010). La cuestión es que las diferencias encontradas en el cerebro de mujeres y hombres no demuestran que son determinantes a la hora de marcar una diferencia en la cognición. Hay diferencias en los cerebros de hombres y mujeres, pero estas no confirman ni avalan determinismo en la cognición ni en el comportamiento. De las diferencias anatómicas y fisiológicas muchos estudios pretendidamente basados en evidencias científicas deducen, de forma ilegítima, diferencias cognitivas y actitudinales. Entre los estudios divulgativos más llamativos y más conocidos estarían los de la neuropsiquiatra estadounidense Louann Brizendine (The Female Brain, 2006; The Male Brain en 2010). Las tesis de esta autora han sido enormemente contestadas (Fine, 2010) en el sentido que sus conclusiones en relación a lo cognitivo y a las diferencias psicológicas y de comportamiento no se derivan de las diferencias neuronales que puedan indicar. Y esto precisamente es lo que podemos tachar de neurosexismo, en el sentido que implica que en las neurociencias, al igual que en otras ciencias, puede estar jugando un papel principal en el diseño de experimentos, en el desarrollo del mismo y en las interpretaciones y conclusiones los prejuicios de género adquiridos en las culturas patriarcales.

Veamos algunos ejemplos:

a) Aptitudes lingüísticas. En el libro The female brain (2006), su autora, Louann Brizendine, concluyó que las mujeres pronuncian 20 mil

5 El meta-análisis o estudio con meta-datos es un método estadístico para agregar resultados de investigaciones científicas a través de muchos estudios sobre el mismo tema o cuestión. Como Janet Shibley Hyde nos demuestra en sus trabajos $(2005,2006,2007)$ es un método ideal para sintetizar la investigación sobre las diferencias en el cerebro entre mujeres y hombres. 
palabras por día, mientras que los hombres sólo 7 mil. Y no sólo eso, sino además de la profusión de palabras, afirmó que las mujeres hablan más rápido. Este estudio fue realizado con una muestra de 5 niñas y 5 niños. Sin embargo, y pese a un defecto grave con la infra-representación de la muestra del experimento, llegó a los medios con titulares tales como: «Hallan la causa de la verborrea femenina» $(A B C, 22 / 02 / 2013)$, o «Cerebro de mujer» (El País, 28/1/2007). El estudio de Brizendine afirma, a partir de las diferencias en el número de palabras emitidas, que las mujeres están mejor preparadas para la comunicación, la empatía y la percepción de las emociones; mientras que los varones lo están, en cambio, para la acción y más pensamiento racional. La autora, no sólo llega a conclusiones universales sobre cerebros femeninos y masculinos, sino que realiza una traslación del supuesto estudio del cerebro en el área del lenguaje a conclusiones comportamentales y actitudinales; como que las chicas tienen más anhelo de relacionarse y de tener «intimidad verbal» (Brizendine, [2006] 2007: 68-69). ¿Cuál es la base científica de esta afirmación? Esto mismo le preguntó a Brizendine el especialista en fonética Mark Liberman (2006), quién buscó las fuentes de esta conclusión y no las encontró. Brizendine reconoció su error y tuvo que eliminar las cifras de ediciones posteriores de su libro. Como el mismo Liberman (2006) relata en su blog lo que sí encontró son estudios que demuestran que no hay diferencia sexual en la aptitud lingüística ${ }^{6}$.

b) Diferencias en cognición moral. El estudio de las diferencias morales en la cognición moral dio lugar a un artículo en 2008 publicado por Harenski et al. (2008) con el título de «Gender differences in neural mechanisms underlying moral sensitivity». Este está dirigido a investigar las regiones del cerebro implicadas en el juicio moral y a ver diferencias de género en la sensibilidad moral a través de resonancia magnética funcional (fMRI). El estudio se realizó con 14 mujeres y 14 hombres. Les escanearon mientras miraban fotos, la mitad de las cuáles mostraban violaciones morales (como un hombre agrediendo a una mujer con un cuchillo). En cada intento se mostraba una foto durante 6 segundos, en los que el/la participante tenía que decir si la foto representaba una violación moral en escala de 1 (no) a 5 (sí y severa). Los resultados fueron que la actividad cerebral durante la sesión de visión de las fotos no mostraba diferencias entre hombres y mujeres. Sin embargo, la actividad cerebral en diferentes niveles sí mostraba diferencias cuando los sujetos clasificaban el grado de violación moral. Las mujeres mostraban

6 Podemos citar el estudio de Matthias R. Mehl, Simine Vazire, Nairán Ramírez-Esparza, Richard B. Slatcher, James W. Pennebaker (2007) que con el título «Are Women Really More Talkative Than Men?», y publicado por la revista Science, presenta un estudio con 396 participantes en el que la conclusión es que no hay diferencias por sexo en la cantidad de palabras que emitimos a diario (la media para los dos sexos es de alrededor de 16.000 palabras). 
más actividad en áreas cerebrales asociadas con las emociones (cingulado posterior y anterior, insula anterior), mientras que los hombres mostraban más actividad en regiones implicadas en el procesamiento cognitivo (sulcus temporal superior). Los autores concluyeron que sus datos son consistentes con la creencia de que las mujeres tratan los dilemas morales con una orientación basada en el cuidado, mientras que los hombres tienden a adoptar evaluaciones morales basadas en la justicia (como ya dijo Carol Gilligan en 1982).

Como la neuróloga del Instituto Pasteur, Catherine Vidal (2011: 300), nos alerta, estas declaraciones, cuando son difundidas al público llevan a creer que los hombres son más confiables que las mujeres en juicios morales porque tienen un cerebro más racional, «como dice la ciencia».

¿Cómo retar esta interpretación que nos parece una conclusión no legítima del experimento? Según C.Vidal (2011: 300) hay que dejar claro tres cosas:

- Las diferencias en el cerebro de una pequeña muestra de participantes no son significativas estadísticamente. La evidencia ha dejado claro que cuando hay un número grande de sujetos analizados las diferencias de sexos normalmente desparecen debido a la gran variabilidad inter-individual del funcionamiento del cerebro.

- Los hallazgos han sido obtenidos en un contexto artificial de laboratorio, el cual no puede ser extrapolado a las experiencias reales de juicios morales.

- La visualización de datos de fMRI sólo provee una imagen parada del estado puntual del cerebro de un individuo. No da evidencia directa sobre los factores biológicos o de los procesos socioculturales que han influido ese estado. Ver las diferencias sexuales en la estructura o función del cerebro no implica que las diferencias existan en el nacimiento ni que vayan a estar grabadas en el mismo a lo largo de la vida.

c) Conexión entre los hemisferios. Recientemente se publicó en la revista Proceedings of the National Academy of Sciences (PNAS) un estudio que, según sus autores, revela una diferencia entre sexos no conocida hasta ahora: la estructura de conexión dentro del cerebro (Ingalhalikar et al., 2014). En su estudio titulado «Sex differences in the structural connectome of the human brain», la experta en análisis de imágenes biomédicas Madhura Ingalhalikar y colaboradores llegan a la conclusión de que los cerebros de personas identificadas como del sexo femenino tienen una mayor conexión entre hemisferios, mientras que los cerebros de las personas identificadas como pertenecientes al sexo masculino muestran mayor conectividad dentro de cada hemisferio. El estudio con tecnología de resonancia magnética, diffusion tensor imaging, fue aplicado a 949 jóvenes de 8 a 22 años, 428 hombres y 521 mujeres. Según los autores del estudio, sus resultados sugieren que los cerebros de personas de sexo masculino están estructurados para facilitar la conexión entre 
percepción y acción, mientras que los cerebros de las personas de sexo femenino están diseñados para facilitar la comunicación entre los procesos analíticos e intuitivos. Resulta significativo, como Ayala, Belli y Broncano señalan (20014: 4), que Ingalhalikar y colaboradores utilicen la expresión "estar diseñado» para referirse a la estructura del cerebro, cuando en el mismo estudio afirman que las diferencias aparecen en la adolescencia (que ellos identifican con la edad de los 13 a los 17 años) y la juventud (entre los 17 y los 22). ¿Qué pasa en los años anteriores? ¿Podrían esas diferencias observadas ser el resultado de una educación diferenciada por sexos? A pesar de que la interpretación más común de este estudio ha sido que hombres y mujeres son diferentes por naturaleza, y así se ha trasladado a los medios de comunicación, el estudio en sí no aporta evidencia para semejante conclusión: ese «diseño» diferenciado por sexos podría ser el diseño que nuestra educación y nuestra cultura sexista nos impone desde que nacemos. Un contexto cultural que precisamente, y por la diferencia de socialización de género, incentiva a los niños a ser competitivos y a las niñas a cooperar.

Como Ayala, Belli y Broncano (2014) señalan, podemos indicar aquí algunos posibles sesgos metodológicos inherentes a trabajos que establecen correlaciones entre diferencias observables en los cerebros, por un lado, y diferencias sociales, por otro. Como ya hemos indicado, esta traslación del cerebro al comportamiento puede ser uno de los pasos que en sí contaminan el mismo proceso científico. Sin embargo, en el artículo original de Ingalhalikar et al. (2014) no aparece información alguna sobre el contexto social y cultural de los participantes, sino únicamente información sobre la raza.

En definitiva: de nuevo podemos hablar de conclusiones no científicas, o pseudo-científicas, y en todo caso, de ciencia alimentada y distorsionada por la cultura sexista.

\section{LA TESIS DE LA NEURO-PLASTICIDAD}

El primero que acuñó el término «plasticidad cerebral» fue el filósofo William James en su libro Principles of Psychology, en 1890. Lo que James introdujo fue la capacidad de organizarse, remodelarse y cambiar del cerebro humano. A esta posibilidad de cambio es a lo que se refiere precisamente el término de «plasticidad cerebral», la cual se entiende que puede ocurrir a nivel de cambios individuales en las neuronas, o a escala de todo el cerebro, tal como ocurre en el remapeo cortical como respuesta a daños cerebrales (Pascual-Leone, et al., 2005, y 2011).

Para nuestro objetivo lo importante será señalar la aportación fundamental de este concepto de neuroplasticidad, y que es la idea de que el cerebro humano no está enteramente "cableado» (hard wired) con circuitos neuronales fijos. Por 
el contrario, esta tesis afirma que hay muchos ejemplos de recableado cortical y subcortical en los circuitos neuronales en respuesta al entrenamiento o a respuestas a daños cerebrales. La neurogénesis, o nacimiento de células cerebrales, se acepta cada vez más como una evidencia que ocurre en los cerebros de mamíferos en la vida adulta y que tal capacidad neurogenerativa puede darse a lo largo de toda la vida (Rakic, 2002; Ponti, et al., 2008) ${ }^{7}$. Lo que podemos extraer de la tesis de la neuroplasticidad para nuestro tema de análisis en este artículo se relaciona con el potencial de la educación, la formación y el entrenamiento de abrir las posibilidades para que nuestros cerebros puedan acomodar individuos y sociedades mejores: más justas, más igualitarias y libres. La idea, no es tanto, como la neurofilosofía de Churchland (1986) nos diría, conocer el cerebro para ver qué podemos esperar de él, qué nos dice y cómo explica nuestras conductas humanas; sino más bien ponernos de acuerdo en cuáles son los valores y prácticas que necesitamos entrenar para que nuestro cerebro active aquellas regiones que permitan a la vez formar individuos y experiencias más significativos para un futuro más humano. La cuestión será organizar un conjunto de valores y un sistema educativo capaz de afectar esa plasticidad y aprovecharse de ella en línea con los valores y aptitudes que de forma consensuada elijamos como humanamente los mejores.

Los estudios evolutivos sobre grupos pueden hacer recaer, como a veces hacen, las diferencias sexuales entre hombres y mujeres en las diferencias adaptativas y funcionales y ello acaba legitimando también cierta desigualdad de funciones y comportamiento. Por ello entender que el cerebro es social, evolutivo y plástico ha de ir de la mano de la idea de que la individualidad de cada cerebro en su plasticidad no se puede explicar del todo por la comunidad de cerebros que podamos plantear (sean estas «mujeres» u «hombres», «negros» o «blancos», o «heterosexuales» u «homosexuales»). La idea, desarrollada por Hyde (2007) en sus investigaciones, es que el género no sólo es una variable diferencial de los individuos, sino una "variable de estímulo», en el sentido de que, tanto en experimentos de laboratorio, como en la vida real, el género de un individuo actúa como un estímulo que influye las respuestas de las personas, así como las respuestas de los otros hacia una persona. Por ello, podemos afirmar que la variación individual obstaculiza la búsqueda de variaciones de grupos discretos. Los estudios realizados con 46 meta-análisis por Hyde (2005) demuestran la hipótesis de la similitud de los cerebros en relación al género. Esta autora concluye que las diferencias de género varian substancialmente en magnitud en las diferentes edades de una persona y dependiendo del contexto en el que se hacen las mediciones. Si tenemos en cuenta que el $90 \%$ de las conexiones neuronales son producidas después del nacimiento (Rakic, 2002),

7 En este sentido son muy conocidos y citados los trabajos de Eleanor MAGUIRE (2000) con los taxistas de Londres; y los de Thomas Elbert (1995) con violinistas diestros obligados a tocar con la mano izquierda. Los experimentos del español Álvaro PAscual-Leone (2005, 2011) son considerados relevantes para señalar cómo esa neuroplasticidad no sólo se activa con la práctica, como en los dos experimentos señalados, sino también con la imaginación. 
ello querrá decir que esas conexiones van a estar en relación con la experiencia y el aprendizaje a lo largo de la vida. En este contexto, parece mucho más razonable considerar que el cerebro no es tan profético, sino que es más bien preparatorio de la funciones (Gergen, 2010: 806-807). Es decir, es un órgano específicamente preparado para la acción, para aprender, para responder continuamente, innovando, según las condiciones de vida que se desarrollen con el tiempo. Es precisamente en este punto que un enorme cuerpo de evidencia de plasticidad neural se vuelve relevante (Gergen, 2010).

Como vemos, la investigación sobre la plasticidad del cerebro, junto con el potencial humano de los cambios en el comportamiento, sugiere que el cerebro facilita más que determina la conducta humana. La determinación, tal y como ya sostuvo Albert Bandura (1925) hace casi un siglo, es mutua entre el cerebro y el ambiente social que nos rodea. La evolución humana es así una evolución bio-cultural o bio-social. Por ello, el entendimiento del ser humano debe estar basado en una relación entre las ciencias, y no tiene sentido llevarla a cabo desde una sola disciplina aislada. El concepto de plasticidad nos da una perspectiva de la biología que trasciende el determinismo neurogenético que implica que la estructura precede a la función. La plasticidad reta, así, las viejas dicotomías de naturaleza y cultura (C. Vidal, 2011: 3).

En cuanto a nuestra cuestión inicial sobre las diferencias sexuales del cerebro podemos afirmar que en gran parte son los estereotipos de género los que a través de la educación a todos los niveles, la socialización y la presión social mantienen las diferencias culturales entre hombres y mujeres; y a su vez, y debido a la plasticidad cerebral, estas diferencias pueden estar modulando y diferenciando las respuestas neuronales. Por ello, y para poder valorar las diferencias individuales en la riqueza que la capacidad plástica y cambiante del cerebro nos permite tendríamos que poder liberarnos de los estereotipos de género. Podemos decir que pueden ser esos prejuicios y roles, entendidos de manera acrítica como diferencias sexuales, los que salvaguardan la realidad de desigualdad que aún hoy, pese al avance en derechos, tenemos entre mujeres y hombres. Hemos visto cómo los estudios científicos sobre las diferencias cerebrales entre hombres y mujeres van reduciendo las diferencias en los resultados cuantos más sujetos tiene el estudio en concreto. Entonces se van borrando las diferencias entre los sexos y van apareciendo más visibles y más significativas las diferencias individuales. También resulta significativo que a mayor educación y cultura más habilidades y competencias tenemos, independientemente si esas habilidades están supuestamente en correlación con nuestro sexo o no (C. Vidal, 2011).

La verdad es que cotidianamente nos resulta muy complicado aceptar explicaciones, por más científicas que sean, de que no hay diferencias esenciales innatas entre hombres y mujeres en relación a sus capacidades mentales, actitudinales, morales. Y ello es así porque nuestras creencias vitales, aprendidas a lo largo de generaciones, y legitimadas por todos los poderes, dan sentido a la forma en que hemos aprendido a entender la vida. Sin embargo, por más difícil que sea retar nuestras creencias, por más cómodos/as que nos sintamos 
con ellas ello no las convierte en innatas. Precisamente ese es el debate entre naturaleza y cultura. La cuestión es si somos conscientes de que las diferencias entre hombres y mujeres, por la evidencia que tenemos, es una cuestión más cultural que biológica; y por ello es prioritariamente una cuestión de género y no de sexo.

\section{CONCLUSIONES: HACIA UNA NEUROCIENCIA CRÍTICA}

La investigación creciente del cerebro humano ha conllevado una progresiva idea de que el sujeto moderno está principalmente localizado en el cerebro. El «sujeto cerebral», como lo denomina F. Vidal (2009), o el «yo neuro-químico», como lo llama Nikolas Rose (2006), se constituye así en la base moderna de la identidad individual. Sin embargo, y a la vista de la falta de evidencias científicas contundentes, ese sujeto cerebral es un prerrequisito de la investigación neurocientífica, y no una conclusión. Esa idea es una hipótesis que las neurociencias aún no han podido demostrar pero que se ha ido sedimentado en el imaginario colectivo como un conocimiento científico probado, cuando en realidad no lo es. Como nos alerta F. Vidal (2009: 21) la idea «eres tu cerebro» en el escenario actual de profusión del discurso mediático sobre el cerebro y las neurociencias, parece convertirse en una afirmación fáctica, mientras que la expresión «eres tú mismo» se considera una afirmación figurativa. Los grandes intereses económicos comprometidos en la financiación de los mega proyectos Human Brain Project y Brain Initiative, vinculados a la declaración de la década de los noventa como la Decade of the Brain, han hecho emerger un interés creciente de las ciencias por captar subvenciones a través de la investigación del cerebro. Ello ha conllevado una «excesiva» confianza y un aumento de expectativas acerca de las posibilidades reales de poder explicar al ser humano completamente a través de una descripción de su cerebro. Sin embargo, y como el mismo Roger W. Sperry alertó cuando recibió el premio Nobel en 1981 por su investigación sobre la especialización funcional de los hemisferios del cerebro, los neurocientíficos deben cambiar sus prioridades y enfatizar los posibles beneficios sociales de sus investigaciones (Sperry, 1981).

Se habla del sujeto como «sujeto cerebral» y la explicación va encaminada a elaborar todas las implicaciones del término cerebral; sin embargo, hay implicaciones también del término «sujeto». Son implicaciones que cuentan con una larga historia y con un debate que prácticamente es el debate filosófico de más impacto en el siglo xx, desde la escuela de Frankfurt, hasta Foucault y más recientemente toda la teoría feminista.

La idea de un sujeto cuya identidad radica fundamentalmente en su cerebro ha suscitado, según nos explica Rose, $(2007,2008)$, el interés en las neurociencias por trazar mapas de lo que se puede llamar la «subjetividad cerebral», «individualidad somática» o "yo neuroquímico» (Rose 2008), «sujeto cerebral» (F. Vidal, 2009) u «hombre neuronal» (Malabou, 2008). El peligro de una vigilancia 
por parte de las ciencias médicas, psicológicas, neurociencias, psiquiatría o biología es clara; y no sólo en cuanto a normativización del cuerpo, sino en la utilización de este conocimiento en ámbitos como la salud, la educación o el derecho, por nombrar algunos ejemplos. El peligro de lo que Foucault ${ }^{8}$ (2001), ya en una de sus clases en 1976, llamó el poder de la bio-política es convertir la vida misma en objeto del poder, con afán de administrarla y gobernarla. Con ese afán se despliega todo un mosaico de herramientas y tecnologías disciplinares que posteriormente se han englobado en el término "bio-gobernanza» (Gerlach et al, 2011). La posible reducción (y determinismo) que puede conllevar la idea de que nuestra identidad reside básicamente en el cuerpo, o parte de él, es, por todo ello, no sólo epistemológicamente impactante, sino políticamente relevante. En especial lo es desde el punto de vista de la teoría feminista, la cual se ha dedicado en profundidad desde su nacimiento a estudiar y comprender por qué y cómo las mujeres hemos sido reducidas a anatomías vulnerables asociadas a estereotipos femeninos. Por ello, y para el tema que nos ocupa, queremos apostar por lo que Kraus (2011) denomina «neurodiversidad» y que refiere a la idea de que hay una variación natural entre los cerebros, que esa variación es inherentemente buena para la humanidad y que las sociedades no sólo tendrían que comprender esto, sino valorarlo como un activo. Este tipo de miradas críticas se sitúan en lo que Kaiser y Dussauge (2015) denominan discursos «no neuro-normativos», los cuáles apelan a una mirada crítica y transdisciplinar a lo que el cerebro es.

Lo que nos descubre una mirada crítica es que la desigualdad no es innata, ni biológica, sino que está construida social y culturalmente sobre la diferencia sexual. La teoría feminista ha demostrado de manera contundente cómo el género construye sobre una diferencia sexual biológica una diferencia social y cultural que conduce a una flagrante desigualdad entre los sexos. Los análisis de la diferencia sexual biológica, (y muy especialmente los referidos al cerebro) que se suponen a la base de este proceso que lleva a la desigualdad suelen estar, como hemos visto, plagados de prejuicios o asunciones que ya de sí contienen precisamente como punto de partida esas ideas de género diferenciado (y desigual). Por ello, no es fácil, en la maraña de investigaciones y publicaciones sobre el tema de la diferencia sexual en el cerebro, acercarnos sin desconfianza ni aprensión a estos estudios. Del análisis de experimentos que hemos visto más arriba hemos podido constatar que en las conclusiones de los mismos hemos detectado actitudes neurosexistas en la exageración de resultados, en experimentos con pocos sujetos y conclusiones no corroboradas; en el uso de expectativas para crear la diferencia sexual y de género que la ciencia no demuestra, convirtiéndose así en una profecía auto-proclamada; o en la creación de neuromitos, que a su vez alimentan y refuerzan los peligros anteriores.

8 El texto en castellano está publicado en 2001, como parte de una colección de sus clases a lo largo de un curso, con el título de Defender la sociedad. Curso en el College de France (1975-1976). La clase en concreto de la que habla de biopoder, si bien después será un concepto en el que profundizará, es la del 17 de marzo de 1976. 
Por todo ello, y para evitar distorsiones como las indicadas y pseudociencia en general, nos parece interesante y necesario llamar la atención sobre la brecha creciente entre los estudios sociales sobre las neurociencias y las neurociencias empíricas (Kraus, 2011). Y así, nos resulta provechosa la propuesta de lo que se ha llamado «neurociencias críticas» (Choudhury, Nagel y Slaby, 2009). Este área de estudio pretende ser un campo de reflexión en el que desde diferentes ámbitos se incida en la auto-reflexividad en las prácticas humanas, y se combine con una exploración de las posibilidades que puedan llevar a las personas afectadas por los desarrollos actuales de las neurociencias a participar en las prácticas y procesos relevantes, para que estos exploren, a su vez, potenciales emancipatorios ${ }^{9}$. Tomando en cuenta las aportaciones de la Teoría Crítica de la Escuela de Frankfurt una ciencia crítica tendrá entre sus intereses generar un conocimiento que permita transformar la realidad para mejorarla, y por ello tenderá a proponer objetivos científicos que puedan conllevar una sociedad más justa y menos opresiva (Hartman, 2012: 69-70). Las neurociencias críticas, por ello, supondrán un compromiso con una perspectiva científica que es conocedora de los intereses prácticos que la investigación científica puede conllevar. La razón esgrimida es la que ya en su día manifestó Horkheimer cuando alertó de que las herramientas conceptuales y metodológicas de la ciencia no pueden estar separadas de los intereses sociales, políticos y económicos (citado en Hartman, 2012: 70). Hartman (2012) sostiene que se trata de una «facticidad normativa» el hecho de que las ciencias, intentando descubrir la primera naturaleza humana, acaban creando la segunda; a base de terminología, metodologías, asunciones y aplicaciones que construyen la interpretación de lo que somos.

Una neurociencia crítica hará uso de un cierto «neuroescepticismo» (Klein, 2011) como estrategia de depuración del conocimiento científico referido al cerebro. La proliferación de neurologismos (Illes, 2009) y neurofilia (Trout, 2008) exige un escepticismo que pueda depurar las afirmaciones que rápidamente pueden ser trasladadas al lenguaje popular de la comunicación de masas sin el rigor científico necesario. El escepticismo, por ejemplo, hacia las inferencias que se pueden hacer de los datos provenientes de las imágenes funcionales del cerebro es necesario, incluso «sistemáticamente necesario», como parte de la evaluación científica de los experimentos (Klein, 2011; Marks, 2010; Roskies, 2008). Podemos entender que por el bien del progreso de la ciencia muchas

9 Como apuntan Choudhury, Nagel y Slaby (2009: 65) la Teoría Crítica de la Escuela de Frankfurt ofrece un adecuado marco para estas Neurociencias críticas. En concreto creen que la noción de «patologías sociales de la razón» explicada por HonNetH en su libro Patologías de la razón: historia y actualidad de la teoría crítica, puede ser un concepto clave para formular tal marco. HARTMANN (2012) también ofrece una crítica a las neurociencias desde la teoria crítica. En esta linea, el trabajo de CoRTina (2013) es de gran relevancia, al ofrecer una teoria que parte de la ética del discurso como un marco adecuado para la neuroética. En su trabajo Cortina señala algunas insuficiencias de ese marco, pero también aporta soluciones para superarlas. 
veces sea bueno ser osados en el planteamiento de hipótesis, pero a la vez, muy conservadores y juiciosos en las conclusiones (Roy, 2007). Además, y como venimos diciendo, habrá que ser muy cautos a la hora de generalizar las hipótesis de manera que sean lanzadas y divulgadas como conclusiones o conocimientos ya probados.

El objetivo de una mirada crítica a las neurociencias es, como ha señalado Marks (2010), transformar la irrealidad que envuelve al discurso neurocientífico acerca de las implicaciones prácticas para el mundo real. Sin embargo, pensamos, que no sólo esa mirada crítica ha de revisar las expectativas del discurso neurocientífico (las inferencias de las técnicas de imágenes funcionales del cerebro, por ejemplo), sino que ha de conectar la investigación científica en sí misma con las consecuencias sociales de la misma. En este sentido, y como reclama Schleim (2014), una neurociencia crítica, si bien es necesaria, no es suficiente ${ }^{10}$. Sería imperioso todo un movimiento de «ciencia crítica» que garantice la autonomía académica y promueva comportamientos responsables con las normas y valores de la investigación científica. Será necesario, así, un marco conceptual que favorezca la colaboración entre las ciencias, así como el compromiso político necesario de todo el conjunto de la producción de conocimiento $^{11}$. Probablemente, y como ha afirmado recientemente Rose (2016), las cuestiones más interesantes a debatir y desvelar no sean tanto técnicas (¿qué neuro-tecnologías harán posible el conocimiento de la mente?), como políticas (¿por qué y para qué ese conocimiento?). En este sentido, una neurociencia simplificadora, sin el necesario diálogo con otras disciplinas, o que de forma prematura traslade el conocimiento científico a la práctica cometerá, usualmente, las confusiones y distorsiones que ya hemos apuntado más arriba, y acabará afirmando que «los hombres son de Marte y las mujeres de Venus» ${ }^{12}$.

\section{Bibliografía}

Ayala, S.; Belli, S. \& Broncanol, F. (2014), «Diferencias, discriminación, cerebro y sexo: controversias científicas de lo social y lo biológico». Encrucijadas. Revista Crítica de Ciencias Sociales, 8: 3-9.

Bem, S. L. (1983), "Gender Schema Theory and Its Implications for Child Development: Raising Gender-aschematic Children in a Gender-schematic Society». Signs 8.4 (1983): 598-616.

10 Por ello, tampoco sería ni aconsejable ni suficiente una posible «neuroética feminista», tal y como SaLLES nos dice (2014).

11 Kraus (2011) mantiene, sin embargo, que un cierto grado de controversia y conflicto es necesario para lograr una reflexividad que precisamente haga a las ciencias comprometerse con asuntos políticos y sociales.

12 Esta frase hace referencia al popular libro de John Gray, publicado originalmente en 1992 por Harper Collins Publishers, el cual ha sido traducido a 50 lenguas y del que se han vendido 50 millones de copias. 
Boddice, R. (2011), «The Manly Mind? Revisiting the Victorian "Sex in Brain” Debate». Gender \& History, Vol. 23 No. 2 August: 321-340.

Bouvaire, S. de [1949] (1998), El segundo sexo (trad. de Alicia Martorell). Cátedra, Madrid.

Brizendine, L. (2006), The Female Brain. Broadway Books, Random House, New York.

Bunge, M. (1985), Pseudociencia e ideología. Alianza Universidad, Madrid.

Butler, J. (1990), Gender Trouble. Routledge. Trad.: El género en disputa. Barcelona, Paidós, 2001.

Choudhury, S.; Nagel, S. K. \& Slaby, J. (2009), "Critical Neuroscience: Linking Neuroscience and Society through Critical Practice». Biosocieties, 4: 61-77. doi:10.1017/ S1745855209006437.

Choudhury, S. \& Slaby, J. (eds.) (2012), Critical Neuroscience: A Handbook of the Social and Cultural Contexts of Neuroscience. Wiley- Blackwell.

Churchland, P. S. (1986), Neurophilosophy: toward a unified science of the mind-brain. Massachusetts Institute of Technology.

Cortina, A. (2013), «Ética del discurso: ¿un marco filosófico para la neuroética?», Isegoría. Revista de Filosofía Moral y Política. N. . 48, enero-junio, 2013: 127-148.

Cortina, A. (2011), Neuroética y Neuropolítica. Tecnos, Madrid.

Cortina, A. (2010), «Neuroética: ¿las bases cerebrales de una ética universal con relevancia política?», en Isegoría, n 42 : 129-148.

DARWIN, C. [1871] (2009), El origen del hombre y la selección en relación al sexo. Alcalé Grupo Editorial.

ELBERT, T. et al. (1995), «Increased cortical representation of the fingers of the left hand in string players». Science 270.

Fausto-Sterling, A. (2000), Sexing the body. Gender politics and the construction of sexuality. Basic Books, New York, NY.

Fine, C. (2010), Delusions of Gender: How Our Minds, Society, and Neurosexism Create Difference. W. W. Norton, New York,

FINE, C. (2008) «Will working mothers' brains explode? The popular new genre of neurosexism», Neuroethics 1 (1): 69-72.

Foucault, M. (2001), Defender la sociedad. Curso en el College de France (1975-1976). FCE, Buenos Aires.

Gergen, K. J. (2010), «The acculturated Brain». Theory \& Psychology 20 (6): 795-816.

Gerlach, N.; Hamilton, S. N.; Sullivan, R.; Walton, P. L. (2011) Becoming Biosubjects: Bodies, Systems, Technologies. University of Toronto Press.

Gilligan, C. (1982), In a different voice: psychological theory and women's development. Harvard University Press, Cambridge, Massachusetts.

Hansson, S. O. (2015), «Science and Pseudo-Science», The Stanford Encyclopedia of Philosophy (Spring 2015 Edition), ZALTA, E. N. (ed.), URL = <http://plato.stanford.edu/ archives/spr2015/entries/pseudo-science/>.

Haraway, D. (1990), Primate visions: Gender, race, and nature in the world of modern science. Routledge, Chapman \& Hall, New York, NY.

Harenski, C. L.; Antonenko, O.; Shane, M.; Kiehl, K. (2008), «Gender differences in neural mechanisms underlying moral sensitivity». Social, Cognitive, and Affective Neuroscience, 3(4): 313-321.

Hartmann, M. (2012), «Against first nature. Critical Theory and Neurosciences», en Choudhury, S. \& Slaby, J., eds. (2012): 67-84.

Honneth, A. (2009), Patologías de la razón: historia y actualidad de la teoría crítica, Katz editores. Original en alemán de 2007. 
Hyde, J. S. (2007), «New Directions in the Study of Gender Similarities and Differences». Current Directions in Psychological Science, 16 (5): 259-263.

Hyde, J. S. (2006), «Gender Similarities Still Rule». American Psychologist, 641-642.

Hyde, J. S. (2005), «The Gender Similarities Hypothesis». American Psychologist, Vol. $60, n^{\circ} 6,581-592$.

Illes, J. (2009), «Neurologisms». The American Journal of Bioethics, 9 (9): 1.

ILLES, J. (2006), Neuroethics: Defining the issues in theory, practice, and policy. Oxford University Press, New York.

James, W. (1890), Principios de Psicología [Principles of Psychology]. Fondo de Cultura Económica, México, 1989.

Joel, D. (2011), «Male or female? Brains are intersex». Frontiers in Integrative Neuroscience, 5 (57): 1-5.

Joel, D. et al. (2015) «Sex beyond the genitalia: The human brain mosaic». Proceedings of the National Academy of Sciences (PNAS), 112 (50): 15468-15473.

Johnson, J. L. \& Repta, R. (2012), «Sex and gender: Beyond the binaries», en Oliffe, J. L. \& Greaves, L. (Eds.), Designing and conducting gender, sex, and health research (pp. 17 -37).Thousand Oaks: Sage Publications.

Jordan-Young, R. M. (2010), Brain Storm: The Flaws in the Science of Sex Differences. Harvard University Press.

KaISER, A. (2012), "Re-Conceptualizing "Sex" and "Gender" in the Human Brain». Zeitschrift fur Psychologie 2012; Vol. 220(2):130-136.

Kaiser, A. \& Dussauge, I. (2015), "Feminist and Queer Repoliticizations of the Brain». EspacesTemps.net, Travaux, 13.04.2015.

KLEIN, E. (2011), «Is There a Need for Clinical Neuroskepticism?». Neuroethics, 4: 251259. DOI: $10.1007 / \mathrm{s} 12152-010-9089-\mathrm{x}$

Kraus, C. (2011), "Critical Studies of the Sexed Brain: A Critique of What and for Whom?». Neuroethics, 5(3): 247-259.

Liben, L. (2016), «We've Come a Long Way, baby (But We're Not There Yet): Gender Past, Present, and Future». Child Development, 87: 1, 5-28.

Liben, L. (2015), «Probability values and human values in evaluating single-sex education». Sex Roles, 72: 401-426.

Liben, L. S. \& Signorella, M. L. (1980), «Gender-related Schemata and Constructive Memory in Children», Child Development, 51 (1): 11-18.

Liberman, M. (2006), «Language Log», Blog del professor Liberman en la web de la Universidad de Pennsylvania. http://itre.cis.upenn.edu/ myl/languagelog/archives/003419.html

Lorber, J. (1996), «Beyond the Binaries: Depolarizing the Categories of Sex, Sexuality, and Gender». Sociological Inquiry, Vol. 66, No. 2, May 1996, 143-159.

LoRber, J. (1993), «Believing is Seen: Biology as Ideology», Gender and Society, 7(4): 568-581.

Maccoby, E. E. \& Jacklin, C. N. (1974), The Psychology of Sex Differences. Standford University Press.

MaguiRe, E. A. et al. (2000): «Navigation-related structural change in the hippocampi of taxi drivers». Proceedings of the National Academy of Sciences (PNAS), 97.

Mahner, M., 2007. «Demarcating Science from Non-Science», pp 515-575, in Theo Kuipers (ed.) Handbook of the Philosophy of Science: General Philosophy of Science - Focal Issues. Elsevier, Amsterdam.

Malabou, C. (2008), What should we do with our brain? Fordham University Press, New York. 
Maney, D. L. (2016), «Perils and pitfalls of reporting sex differences». Philosophical Transactions B. The Royal Society Publishing, Vol.371, n.1688. 2016371 20150119; DOI: 10.1098/rstb.2015.0119

Marks, J. H. (2010), «A Neuroskeptic's guide to neuroethics and national security». American Journal of Bioethics Neuroscience. 1: 4-12.

Medina-Vicent, M. (2016), «Neurociencia y teoría política feminista. La inestabilidad sexo-género-sexualidad a través de la obra de Paul B. Preciado». Pensamiento, 2016.

Mehl, M. R. S. V., Ramírez-Esparza, N., Slatcher, R. B., Pennebaker (2007), «Are Women Really More Talkative Than Men?». Science, 6 Jul 2007: 82.

Mill, John Stuart [1869] (2010), El sometimiento de la mujer. Alianza Editorial.

Organization for Economic Co-operation and Development (OECD) (2007), Understanding the Brain: The Birth of a Learning Science. Centre for Educational Research and Innovation. Paris: OECD Publications (Traducción al español en 2010: La comprensión del cerebro: El nacimiento de una ciencia del aprendizaje).

Organization for Economic Co-operation and Development (OECD) (2002), «Learning seen from the neuroscientific approach». In: Understanding the brain: Towards a New Learning Science. Paris: OECD Publications.

Pallarés-Domínguez, D. (2016), «Neuroeducación en diálogo: neuromitos en el proceso de enseñanza-aprendizaje y en la educación moral». Pensamiento, 2016.

Pascual-Leone, A., Amedi A., Fregni F., Merabet, L. B. (2005), «The plastic human brain cortex». Annual Review of Neuroscience 28: 377-401.

Pascual-Leone, A., Freitas C., Oberman L., Horvath J. C., Halko M., Eldaief M.; et al. (2011), "Characterizing brain cortical plasticity and network dynamics across the age-span in health and disease with TMS-EEG and TMS-fMRI». Brain Topography 24: 302-315.

Ponti, G.; Peretto, P.; Bonfanti, L.; Reh, T. A. (2008), Reh, T. A., ed. «Genesis of Neuronal and Glial Progenitors in the Cerebellar Cortex of Peripuberal and Adult Rabbits». PLOS ONE, 3 (6): e2366.

RAKIC, P. (2002), «Neurogenesis in adult primate neocortex: an evaluation of the evidence». Nature Reviews Neuroscience, 3 (1): 65-71.

Reverter-Bañón, S. (2010), "La deriva teórica del feminismo», DAIMON. Revista Internacional de Filosofía, Suplemento 3: 153-162.

Rose, N. (2016), «Reading the Human Brain: How the Mind Became Legible». Body \& Society, 22(2): 140-177.

Rose, N. (2014), «Five minutes with Nikolas Rose. An interview». En el blog de The London School of Economics and Political Sciences, The Impact Blog, http://blogs. lse.ac.uk/impactofsocialsciences/2014/02/06/five-minutes-with-nikolas-rose/ Consultado el 3 de marzo de 2016.

Rose, N. (2007), «Molecular Biopolitics, Somatic Ethics and the Spirit of Biocapital». Social Theory and Health, 5 (1): 3-29.

Rose, N. (2006), The Politics of Life Itself: Biomedicine, Power, and Subjectivity in the Twenty-First Century. Princeton University Press, Princeton, NJ.

Rose, N. (1998), Inventing Our Selves: Psychology, Power and Personhood. Cambridge University Press.

Rose, N. (1989), Governing the Soul: The Shaping of the Private Self (1st edition). Routledge.

Rose, N. \& Abi-Rached, J. M. (2013), Neuro: The New Brain Sciences and the Management of the Mind. Princeton University Press.

Roskies, A. (2008), «Neuroimaging and inferencial distance». Neuroethics 1: 19-30. 
Roskies, A. (2002), «Neuroethics for the new Millenium». Neuron, 35 (1): 21-23.

Roy, D. (2007), «Somatic Matters: Becoming Molecular in Molecular Biology». Rhizomes. Cultural Studies in Emerging Knowledge, 2007, 14. Publicación online, accessible en: http://www.rhizomes.net/issue14/roy/roy.html.

Runnels, V.; Tudiver, S.; Doull, M. \& Boscoe, M. (2014), «The challenges of including sex/gender analysis in systematic reviews: a qualitative survey». Systematic Reviews. BioMedCentral.3:33.http://systematicreviewsjournal.biomedcentral.com/articles/10.1186/2046-4053-3-33. Consultado el 4 de mayo de 2016.

SalLEs, A. (2014), «Las diferencias sexuales y la discusión neuroética». Debate Feminista, Volume 49: 94-115

Schleim, S. (2014), "Critical neuroscience - or critical science? A perspective on the perceived normative significance of neuroscience». Frontiers in Human Neuroscience, 8, May, 1-6.

Sperry, R. W. (1981), "Changing Priorities». Annual Review of Neuroscience, 4, 1-15.

Springer, K. W; Stellman, J.M.; Jordan-Young, R. M. (2011), «Beyond a catalogue of differences: a theoretical frame and good practice guidelines for researching sex/ gender in human health». Social Science \& Medicine 2011, 74 (11): 1-8.

Trout, J.D. (2008), «Seduction without case: Uncovering explanatory neurophilia». Trends in Cognitive Sciences 12 (8): 281-282.

Vidal, C. (2011), «The Sexed Brain: Between Science and Ideology». Neuroethics, 5(3): 295-303.

VIDAL, F. (2009), «Brainhood, anthropological figure of modernity». History of the Human Sciences, 22(1): 5-36.

Wood, J. (2001), Passion and Pathology in Victorian Fiction. Oxford University Press.

World Health Organization. WHO (2001) ICF: International Classification of Functioning, Disability, and Health. Geneva Who Editions.

Zadetto-Smith, A. M.; Mu, K.; Phelps, C. L.; Houtz, L. E. \& Royee, C. B. (2002) «Brains Rule Fun = Learning = Neuroscience Literacy». Neuroscientist 8(5): 396-404.

Departamento de Filosofia y Sociologia.

SONIA REVERTER-BAÑóN

Universitat Jaume I

reverter@uji.es

[Artículo aprobado para publicación en octubre de 2016]. 